\title{
Aerobic exercise training counteracts insulin MEDICINA resistance induced by low sodium diet by increasing insulin signaling in muscle
}

TSS

$\underline{\text { Paula Ramos Pinto }}{ }^{1}$, V. Del Bianco ${ }^{1}$, A.P. Garcia Bochi ${ }^{1}$, G. Silva Ferreira ${ }^{1}$, M. da Silva Trevisani ${ }^{1}$, L. Gomes Rodrigues ${ }^{1}$, E.R. Nakandakare ${ }^{1}$, M. Okamoto ${ }^{2}$, U. Fabres Machado ${ }^{2}$, S. Catanozi ${ }^{1}$, M. Passarelli ${ }^{1,3}$.

${ }_{1}^{1}$ Faculdade de Medicina da Universidade de São Paulo, Laboratório de Lípides LIM10 - Hospital das Clinicas HCFMUSP, São Paulo, Brazil.

${ }^{2}$ Instituto de Ciências Biomédicas- Universidade de São Paulo ICB-USP, Departamento de Fisiologia e Biofísica, São Paulo, Brazil.

${ }^{3}$ Universidade Nove de Julho, Programa de Pós-Graduação em Medicina, São Paulo, Brazil.

e-mail: paularamos@usp.br

Funding: FAPESP, Brazil (2011/15153-1)

\section{Introduction}

Low sodium diet contributes to reduce cardiovascular disease. However some studies have demonstrated severe metabolic alterations such as plasma hyperlipidemia, insulin resistance, activation of renin-angiotensinaldosterone and sympathetic systems induced by sodium restriction diet.

\section{Aim}

To investigate the effect of aerobic exercise training (AET) in the insulinresistance induced by low-

sodium (LS) diet in LDLRKO mice focusing in the insulin-signaling in skeletal muscle.

\section{Methods}

Three-month old LDLRKO mice were fed a chow normal sodium $(1,27 \%-\mathrm{NaCl} ; \mathrm{NS})$ or a LS $(0,15 \%-$ $\mathrm{NaCl}$; LS) diet, and concomitantly kept sedentary (S-NS or S-LS) or submitted to AET (T; T-NS or T-LS) in treadmill (1h/day, $15 \mathrm{~m} / \mathrm{min}, 5$ times week) during 3 months. Exercise capacity, body weight, blood pressure, plasma cholesterol, triglycerides, fasting glucose and glucose decay rate by the insulin tolerance test (ITT) were determined. GLUT4, total and phosphorylated AKT in whole gastrocnemius extract were analyzed by immunoblot. Comparisons were done by 2-way ANOVA. This study was approved by The Ethical Committee - CEUA Faculdade de Medicina da Universidade de São Paulo (№ 071/16)

\section{Conclusion}

AET prevents the LS diet-induced insulin resistance in LDLRKO mice by enhancing total and phosphorylated AKT and GLUT4 content in skeletal muscle.

With thanks to the EAS for support in he form of Young Investigator Fellowship

\section{Results}

TABLE 1. Body weight, plasma lipids and glucose, blood pressure and exercise capacity in LDLRKO mice after experimental protocol.

S-NS T-NS S-LS T-LS

$p$

$(n=4-13) \quad(n=5-12) \quad(n=4-14) \quad(n=4-12) \quad$ Interaction $\quad$ Diet Exercise

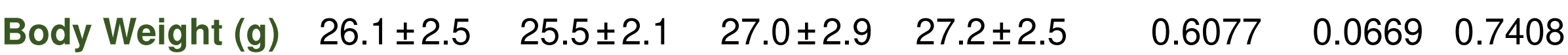

$\begin{array}{llllllll}\mathrm{TC}(\mathrm{mg} / \mathrm{dL}) & 271 \pm 103 & 304 \pm 91 & 254 \pm 96 & 292 \pm 83 & 0.9534 & 0.7721 & 0.4723\end{array}$

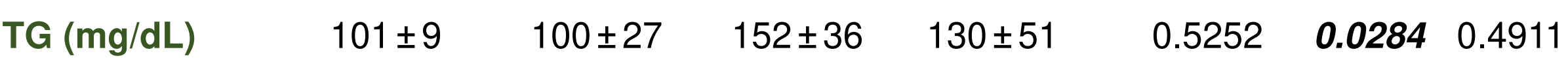

$\begin{array}{llllllll}\text { Glucose }(\mathrm{mg} / \mathrm{dL}) & 101 \pm 16 & 113 \pm 15 & 115 \pm 19 & 124 \pm 23 & 0.7374 & \mathbf{0 . 0 2 2 4} & \mathbf{0 . 0 4 7 7}\end{array}$

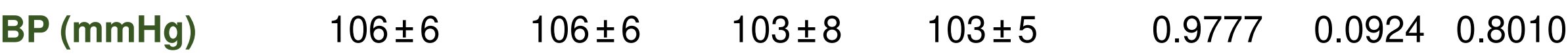

EC (sec) $\quad 1097 \pm 236 \quad 1995 \pm 464 \quad 1053 \pm 244 \quad 1866 \pm 593 \quad 0.7095 \quad 0.4495<0.0001$

Data expressed as mean $\mathrm{SD} ; \mathrm{TC}=$ total cholesterol; $\mathrm{TG}=$ triglycerides; $\mathrm{BP}=$ blood pressure, $E C=$ exercise capacity.

Insulin tolerance test (ITT)

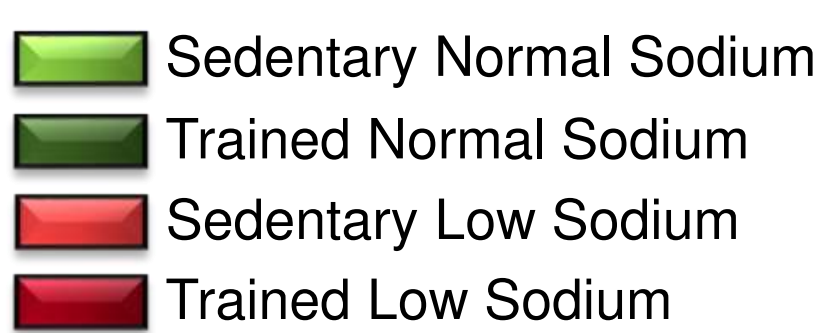

$$
{ }^{*} p<0.005
$$

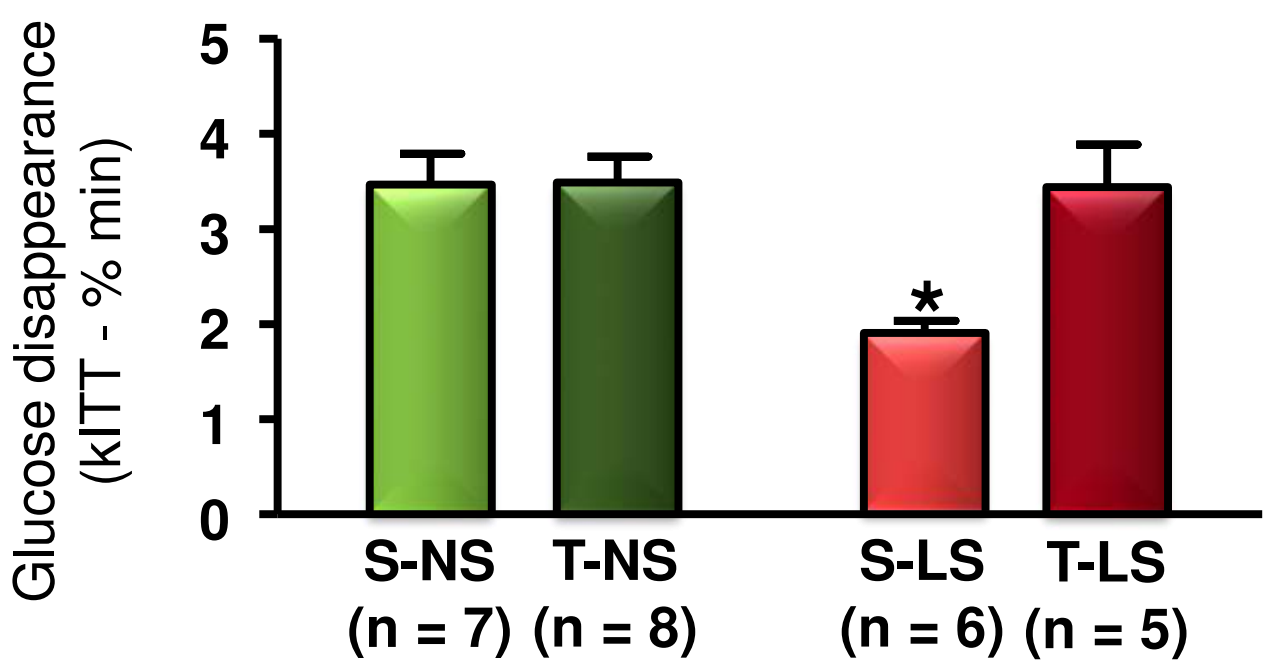

GLUT4, total AKT and phosphorylated protein level in gastrocnemius muscle

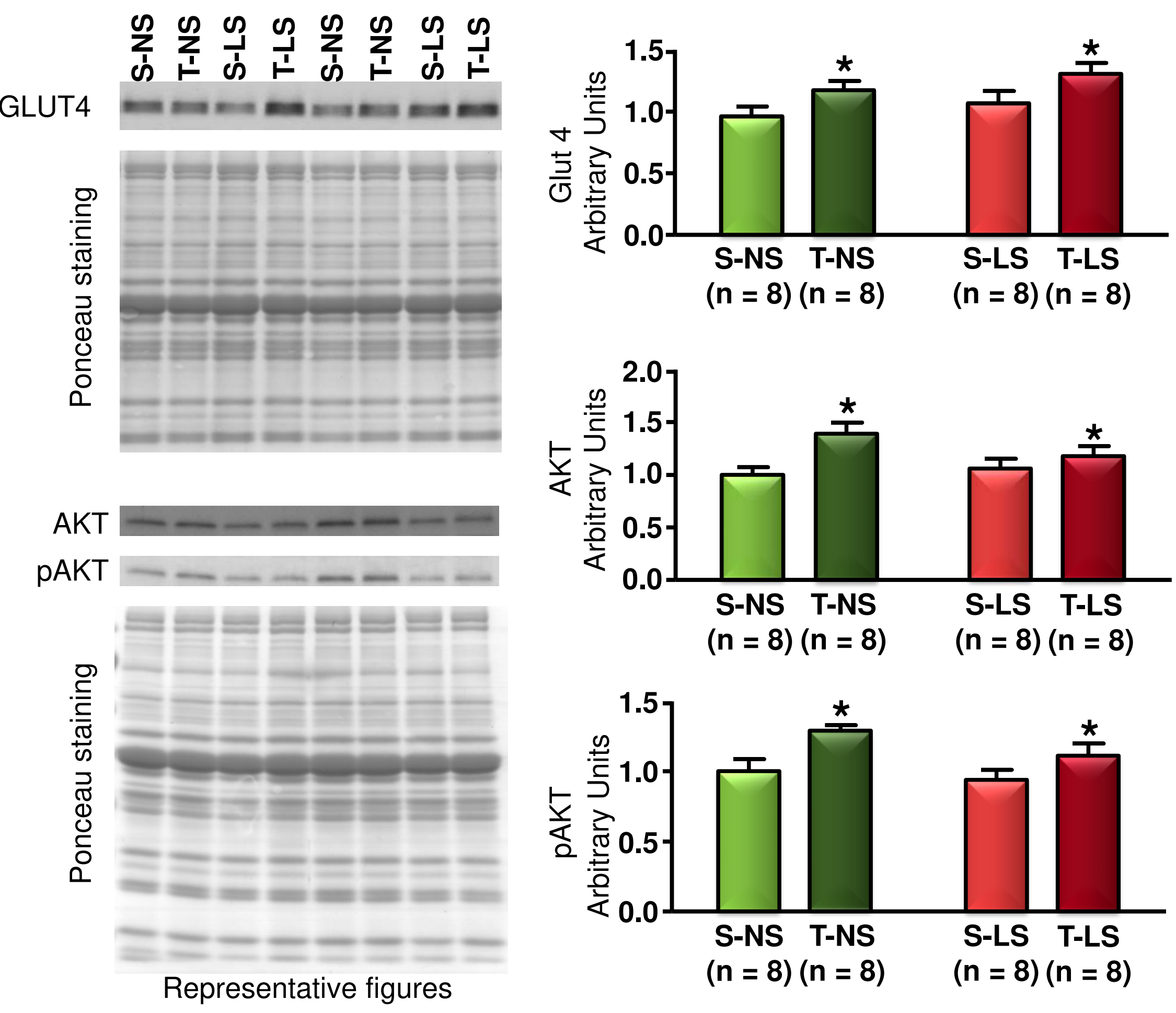

